\title{
RESEARCH
}

Open Access

\section{$\beta$-amyloid induces a dying-back process and remote trans-synaptic alterations in a microfluidic-based reconstructed neuronal network}

Bérangère Deleglise ${ }^{1,2,3,4 \dagger}$, Sebastien Magnifico ${ }^{3,4 \dagger}$, Eric Duplus ${ }^{1,2,3,4}$, Pauline Vaur ${ }^{1,2,4}$, Vanessa Soubeyre ${ }^{3,4}$, Morgane Belle ${ }^{3,4}$, Maeva Vignes ${ }^{3,4,5,6}$, Jean-Louis Viovy ${ }^{5,6}$, Etienne Jacotot ${ }^{1,2,4,7}$, Jean-Michel Peyrin 1,2,3,4 and Bernard Brugg 1,2,3,4*

\begin{abstract}
Introduction: Recent histopathological studies have shown that neurodegenerative processes in Alzheimer's and Parkinson's Disease develop along neuronal networks and that hallmarks could propagate trans-synaptically through neuronal pathways. The underlying molecular mechanisms are still unknown, and investigations have been impeded by the complexity of brain connectivity and the need for experimental models allowing a fine manipulation of the local microenvironment at the subcellular level.
\end{abstract}

Results: In this study, we have grown primary cortical mouse neurons in microfluidic ( $\mu F D)$ devices to separate soma from axonal projections in fluidically isolated microenvironments, and applied $\beta$-amyloid (A $\beta$ ) peptides locally to the different cellular compartments. We observed that $A \beta$ application to the somato-dendritic compartment triggers a "dying-back" process, involving caspase and $\mathrm{NAD}^{+}$signalling pathways, whereas exposure of the axonal/distal compartment to $A \beta$ deposits did not induce axonal degeneration. In contrast, co-treatment with somatic sub-toxic glutamate and axonal $A \beta$ peptide triggered axonal degeneration. To study the consequences of such subcellular/local $A \beta$ stress at the network level we developed new $\mu F D$ multi-chamber devices containing funnel-shaped micro-channels which force unidirectional axon growth and used them to recreate in vitro an oriented cortico-hippocampal pathway. $A \beta$ application to the cortical somato-dendritic chamber leads to a rapid cortical pre-synaptic loss. This happens concomitantly with a post-synaptic hippocampal tau-phosphorylation which could be prevented by the NMDAreceptor antagonist, MK-801, before any sign of axonal and somato-dendritic cortical alteration.

Conclusion: Thanks to $\mu F D$-based reconstructed neuronal networks we evaluated the distant effects of local A $\beta$ stress on neuronal subcompartments and networks. Our data indicates that distant neurotransmission modifications actively take part in the early steps of the abnormal mechanisms leading to pathology progression independently of local $A \beta$ production. This offers new tools to decipher mechanisms underlying Braak's staging. Our data suggests that local A $\beta$ can play a role in remote tauopathy by distant disturbance of neurotransmission, providing a putative mechanism underlying the spatiotemporal appearance of pretangles.

Keywords: Microfluidics, Neuronal network, Axonal degeneration, Tau, Synapse loss

\footnotetext{
* Correspondence: bernard.brugg@upmc.fr

${ }^{\dagger}$ Equal contributors

'Institut de Biologie Paris Seine (IBPS), 75005 Paris, France

${ }^{2}$ CNRS UMR 8256, Biological Adaptation and Ageing, Team 5: Degenerative

Processes in Neurons and Networks, F-75005 Paris, France

Full list of author information is available at the end of the article
} 


\section{Introduction}

The two main hallmarks of Alzheimer's disease (AD) are extracellular deposits composed of $\beta$-amyloid peptide (senile plaques) and intracellular filamentous aggregates composed of self-assembled hyperphosphorylated Tau proteins (neurofibrillary tangles, NFTs). Histopathological studies show that these hallmarks spread, each in their own stereotyped fashion, within specific regions of the brain during disease evolution [1,2]. This progression follows neuro-anatomical pathways and could be the sign of nexopathy-related processes [3]. A large body of evidence indicates that neurons affected in $\mathrm{AD}$ follow a dying-back pattern of degeneration, where abnormalities in synaptic function and axonal integrity long precede somatic cell death $[4,5]$. Since neurons are highly polarized, this raises the question whether local $A \beta$ and Tau protein abnormalities in the vicinity of different neuronal subparts (somatic, dendritic, axonal) lead to local degenerative processes or could initiate distant dysfunction within neurons or even within neuronal networks, through (trans-) synaptic alterations. However, though various mechanisms initiating local primary dysfunctions have been described, the molecular or structural reasons underlying distant breakdown of specific networks and the relationship between amyloid and Tau pathology are still unclear.

Recent advances in the development of microfluidic devices $(\mu \mathrm{FD})$ facilitate microenvironment development adapted to neuronal structures and subdomains with easy access and control $[6,7]$. We have previously designed and developed a $\mu \mathrm{FD}$ system to polarize neuronal networks using funnel-shaped micro-channels, also called axonal diodes, allowing the reconstruction of an orientated functional cortico-striatal neuronal network in vitro [8]. In the present study, we used a similar $\mu$ FD-based approach to study the molecular mechanism of neurodegenerative processes in compartmentalized neurons and neuronal networks. By compartmentalizing axon terminals from cell bodies of cortical neurons, we demonstrate that axons are partially resistant to axonal $\mathrm{A} \beta$-peptide exposure, while somatic treatments trigger an anterograde degeneration signal in axons, inducing a dying-back pattern. We then reconstructed an oriented cortico-hippocampal network in $\mu \mathrm{FD}$, and showed that somato-dendritic deposits of $\mathrm{A} \beta$ on cortical neurons trigger a rapid cortical presynaptic disconnection concomitant with a glutamate-dependent postsynaptic hippocampal tau-phosphorylation before any axonal and somatic cortical degeneration.

\section{Results}

\section{Somato-dendritic exposure of cortical neurons to} $A \beta$-peptide induces an axonal dying back pattern

We wondered whether localized $\beta$-amyloid deposition on different subcellular compartments (somato-dendritic or axonal) lead to local degenerative signals or to a global neuronal degeneration. Axons were compartmentalized from the somato-dendritic compartment by seeding cortical neurons in $\mu \mathrm{FD}$ devices comprising two chambers connected through asymmetrical micro-channels ( $2 \mathrm{C}-\mu \mathrm{FD}$ diodes). When seeded in the left chamber, cortical neurons projected their axons from the somato-dendritic compartment to the distal compartment through filtering micro-channels (Figure 1a-a,b). Analysis of dendrites (MAP2, red) and nuclear chromatin visualization (DAPI, blue) indicated that the seeded cortical neurons were healthy after two weeks in culture (Figure 1b, Additional file 1: Figure S1a). Somato-dendritic application of fibrillar A $325-35$, mimicking amyloid plaques, induced axonal degeneration process (Figure 1c, g). While somato-dentritic application of aggregated $A \beta$ did not affect somatic and dendritic viability (MAP2, red; DAPI, blue; Figure 1c) some local synaptic damage was evidenced in the somatic chamber (MAP2, blue; Vglut1, red; Additional file 1: Figure S1b). Addition of $\mathrm{NAD}^{+}$, pharmacological inhibitors of c-Jun N-terminal kinases (JNK, SP600125), or caspases inhibitor (z-VAD-fmk), to the axonal compartment significantly reduced $A \beta$-induced axonal degeneration (Figure 2). In contrast, distal axonal application of aggregated $A \beta$ failed to induce any significant axonal degeneration in our paradigm (Figure 1d-g). Interestingly, while low dose of somato-dendritic glutamate treatment did not trigger axonal degeneration per se (Figure 1e-g, tubulin, grey), the combination of distal $A \beta$ application with this sub-toxic dose of glutamate to the somato-dendritic compartment triggered a massive axonal fragmentation (Figure 1f-g). This suggests that axonal resistance toward local $\mathrm{A} \beta$ deposition is tightly controlled by the somato-dendritic behavior and that upstream neurotransmitter dysfunction may precipitate neuronal network collapse.

\section{Cortical somato-dendritic $\beta$-amyloid peptide exposure induces a rapid disconnection of cortico-hippocampal synapses, which precedes axonal degeneration}

To decipher whether local $A \beta$ might induce remote toxic effect on synapses we used $2 \mathrm{C}-\mu \mathrm{FD}$ "axonal diodes" chips that allows reconstructing oriented neuronal networks [8], to create a unidirectional cortico-hippocampal network. When primary cortical neurons $(\mathrm{Cx})$ were cultured at high density in the left chamber of the device and hippocampal neurons $(\mathrm{Hi})$ were seeded at low density in the right chamber (Figure 3a), cortical neurons projected axons (Figure 3b-c; $\alpha$-tubulin, green) through the funnel-shaped micro-channels and established synapses with hippocampal neurons in the right chamber (Figure 3b-d; MAP2, blue; $\alpha$-synuclein, red). Thanks to the funnel-shaped $\mu$-channels, hippocampal neurons did not project axons backwards. While hippocampal neurons cultured alone showed few presynaptic cluster along their dendritic shaft (insert in Figure $3 \mathrm{~b}$ and $\mathrm{d}$ ), Hippocampal neurons grown in contact 
a

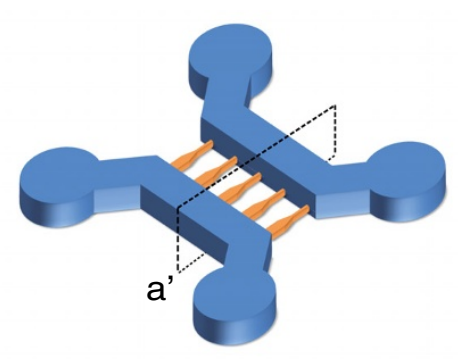

$a^{\prime}$

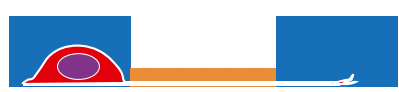

g

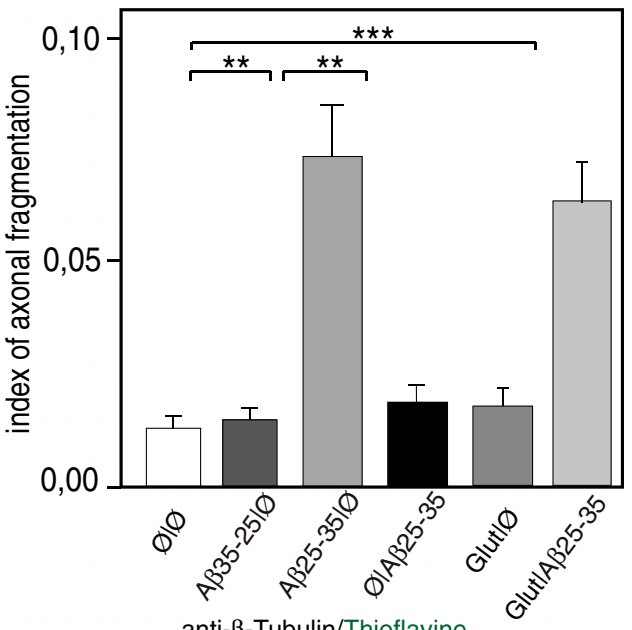

anti-MAP2/Dapi Dapi/Thioflavine

b
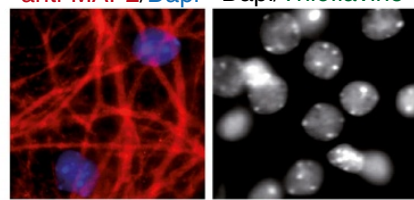

C
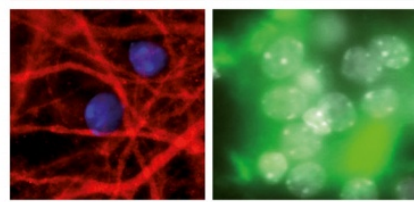

d

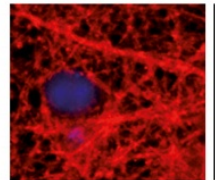

e
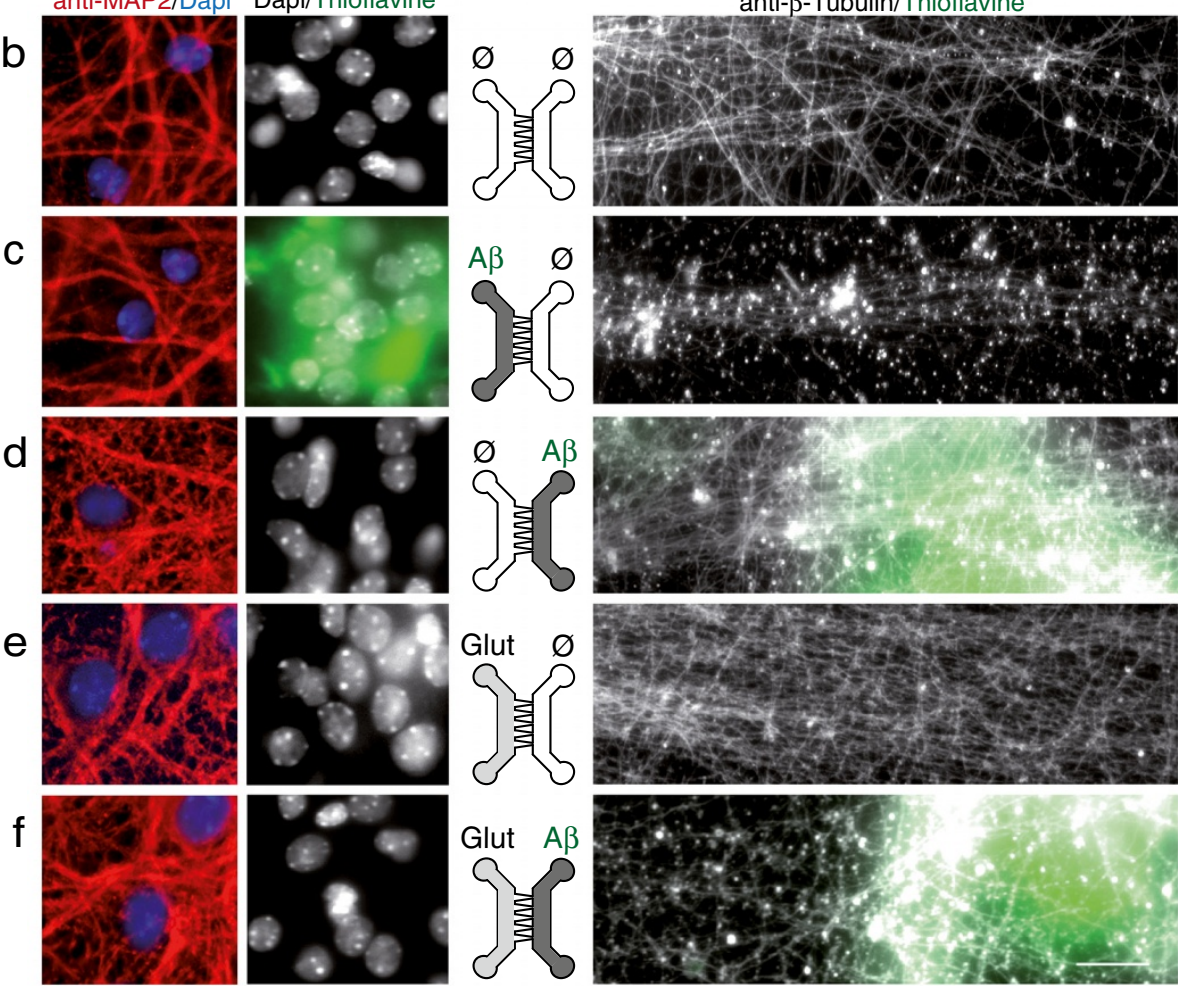

Glut
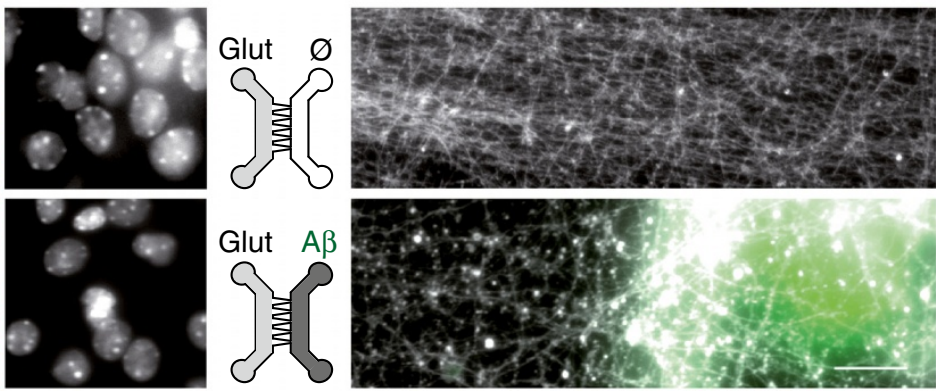

Figure 1 Somatic applications of $\mathbf{A} \boldsymbol{\beta}$ peptides induce a dying-back pattern. $\mathbf{a}$. Schematic representation of a two-chamber microfluidic device (2-C $\mu \mathrm{FD})$ with two cell culture chambers interconnected by funnel-shaped micro-channels [8] (a': cross-section of the microfluidic device. A neuron is represented. Note that only axons can reach the right chamber). b-f. Fluorescence microscopy analysis of axonal degeneration vs somatic status after addition of A $\beta$ peptide in the left (soma) vs right (distal part of axons) chambers. Cortical neurons were grown for 12 days in two-compartment chips and each chamber was treated for $48 \mathrm{~h}$ with $\mathrm{AB}$ peptides as indicated. The two left micrographs show the somato-dendritic chamber after staining with anti-MAP2 (red), DAPI (blue) and Thioflavine S (green). The right rectangular micrographs show the distal chamber after immunostaining with anti-a-tubulin and labeling with Thioflavine $\mathrm{S}$. Each central scheme represents the device with indicated localization of treatment (left chamber vs right chamber): b) control cultures $(\varnothing / \varnothing)$, c) somato-dendritic treatment with $A \beta 25-35$ peptide $(A \beta / \varnothing, 30 \mu M)$, d) axonal treatment with $A \beta 25-35$ peptide $(\varnothing / A \beta, 30 \mu \mathrm{M})$, e) somato-dendritic treatment with glutamate (Glut/ $\varnothing, 10 \mu \mathrm{M})$, f) somato-dendritic treatment with glutamate combined with axonal treatment A $25-35$ (Glut/A, $10 \mu \mathrm{M} / 30 \mu \mathrm{M}$ ). Scale bar: $20 \mu \mathrm{m}$. g) Quantification of axonal fragmentation. A $25-35$, glutamate (Glut), and control Aß35-25 were added, or not (Ø), to the chamber as indicated. Axonal fragmention index was calculated as described in Methods section $\left(n=3 ;{ }^{* *} P<0.01 ;{ }^{* * *} P<0.001\right.$, ANOVA). 


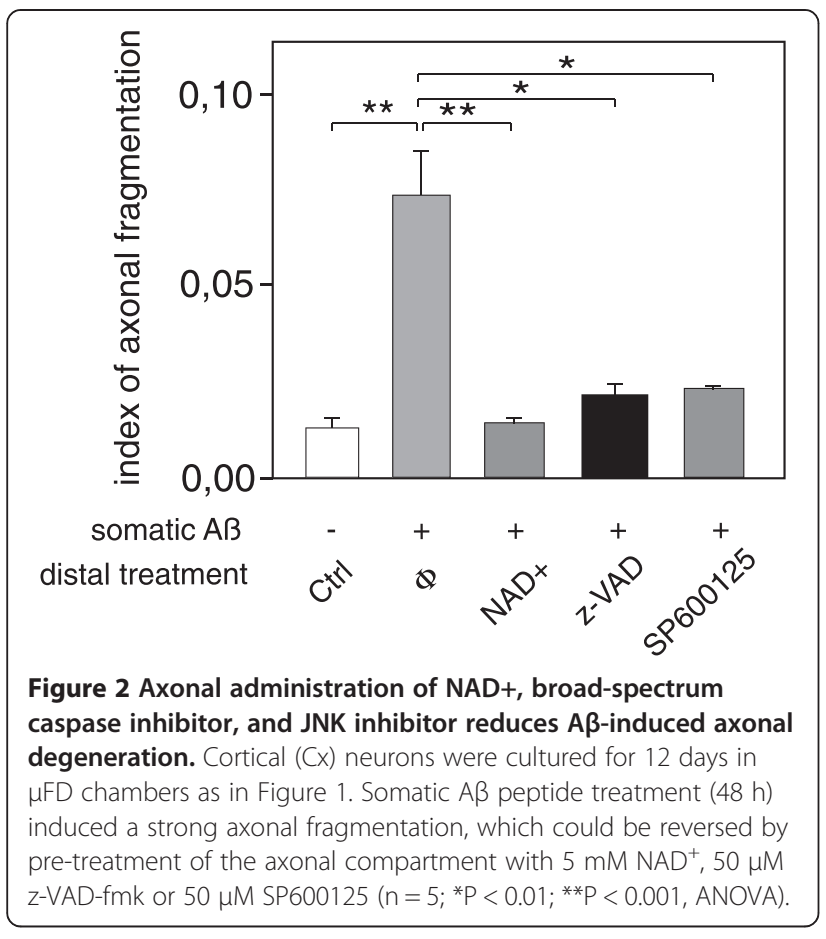

with cortical fibers showed high density presynaptic clusters as evidenced by dense $\alpha$-synuclein (Figure 3d) or VGLUT1 (not shown) staining along the hippocampal dendrites. Application of A $325-35$ peptide to the Cx compartment induced cortico-hippocampal synapse loss in the Hi compartment within $24 \mathrm{~h}$ (Figure 3c-e), whereas cortical axons and soma showed no obvious sign of degeneration (Figure 3c). Similar results were obtained with nanomolar doses of oligomeric or fibrillar $\mathrm{A} \beta 1-42$ (Figure 3e) suggesting that this process does not rely on the aggregation state of the peptides. While cortical fibers were still intact $24 \mathrm{~h}$ after $\mathrm{A} \beta$ (peptide or oligomer) application, at $48 \mathrm{~h}$ after treatment, connected axons started to degenerate (Figure 4). We thus observed that the first structural alteration following somato-dendritic $A \beta$ deposits is a distant synapse loss that is followed by delayed axonal degeneration, reminiscent of a dying back process.

\section{Selective cortical $A \beta$ peptide exposure induces Tau Thr231 phosporylation in a distant fluidically isolated hippocampal neuron through synaptic NMDA receptor-dependent neurotransmission}

It is known that a direct exposure of hippocampal neurons to extracellular soluble $\mathrm{A} \beta$ oligomers induce in vivo and in vitro intracellular Tau hyperphosphorylation $\left(\operatorname{tauHP} \mathrm{P}^{+}\right)$ and trigger decreased microtubule stability and NFT formation [9]. We consequently examined whether $A \beta$ deposits (oligomeric or fibrillar $A \beta 1-42$, or $A \beta 25-35$ ) in the $\mathrm{Cx}$ chamber could induce distant post-synaptic Tau phosphorylation in the Hi chamber. The antibody recognizing the phospho-threonine 231 epitope detects one of the earliest phosphorylation changes observed in $\mathrm{AD}$ patients [10]. After $24 \mathrm{~h}$ of $\mathrm{A} \beta$ treatment in the Cx chamber we found that phosphorylation levels of Tau Thr231 increased in post-synaptic hippocampal neurons (Figure 5; pTau T231, pseudo-colors). Tau Thr231 phosphorylation was particularly concentrated in the cell bodies and proximal dendrites of hippocampal neurons, rather than in distal dendrites and axons. This effect appeared to be glutamate dependent as phosphorylation was prevented by hippocampal pre-treatment with the NMDA receptor antagonist, MK801 (Figure 5e). Hence, $\mathrm{A} \beta$-mediated disturbance of glutamatergic neurotransmission and concomitant synapse loss can induce Tau phosphorylation in connected hippocampal neurons and subsequently axonal degeneration of cortical fibers, and therefore initiates progressive neuronal network collapse (Figure 6).

\section{Discussion}

A large body of evidence indicates that neurons affected in $\mathrm{AD}$ follow a dying-back pattern of degeneration, where such loss of axonal integrity precede somatic cell death and has a profound effect on neuronal network function. However, the molecular mechanism underlying dying-back of neurons and its consequences on the neuronal network in $\mathrm{AD}$ remain elusive and difficult to study in vivo. Using a new $\mu \mathrm{FD}$ system [8], we modeled for the first time the perforant pathway, known to be affected early in $\mathrm{AD}[1,2]$. Somato-dendritic $\mathrm{A} \beta$ cortical application within cortico-hippocampal network leads to a rapid presynaptic collapse before cortical axonal or somatic loss $[4,5]$. Since these synapses were not exposed to $A \beta$, this suggests that local somato-dendritic $A \beta$ deposits have fast remote toxicity on the unchallenged synapses. This could be due either to a self-propagation [11] and to rapid distribution of $A \beta$ through axonal transport [12] or to the induction of a signal in the soma, which is transmitted through the neuron. We recently described similar distant synapto-toxicity following axotomy [13]. Although no short-term morphological alteration of postsynaptic hippocampal neurons was observed, the A $\beta$-induced remote loss of cortical presynapses was concomitant to Tau Thr231 phosphorylation in the interconnected postsynaptic hippocampal neurons, and occurred well before axonal and somatic degeneration of cortical neurons. Thus local $\mathrm{A} \beta$ deposits generate fast propagation of degenerative signals across networks leading to early dysfunctions in remote areas. Our results show that local somato-dendritic (but not axonal) $A \beta$ triggers distal-to-proximal axonal degeneration before any somato-dendritic abnormalities, a process reminiscent of dying-back pattern observed in various neurodegenerative syndromes [14]. Thus $A \beta$ toxicity depends not only 
a

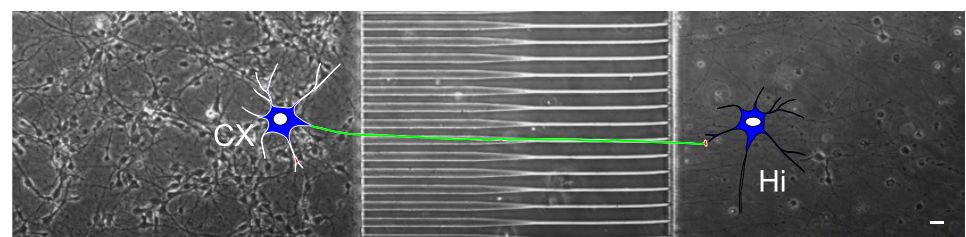

b
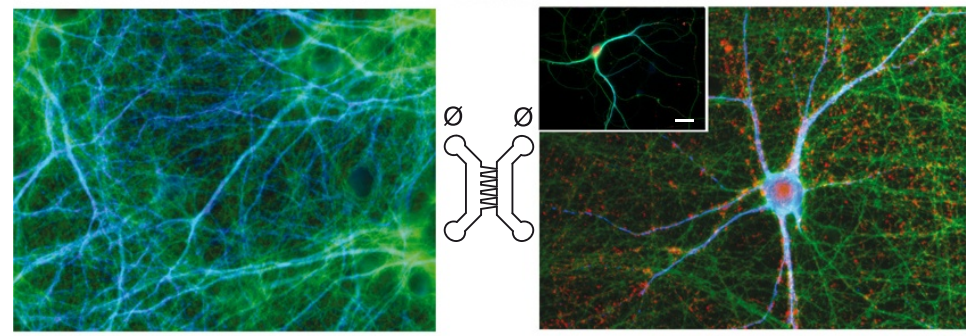

c
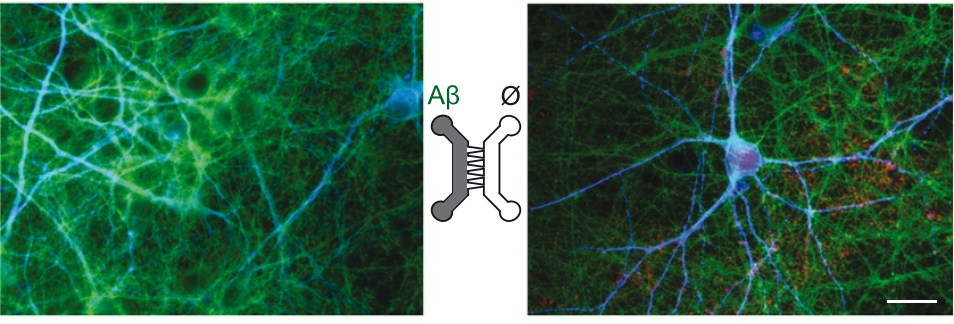

d

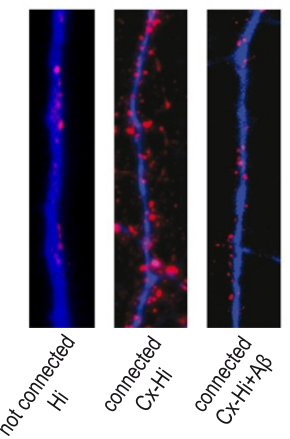

e

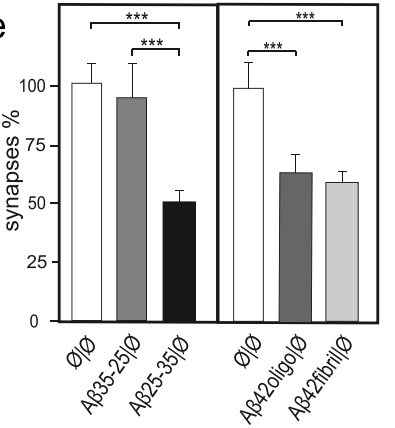

Figure 3 Cortical A $\beta$-peptide exposures induce early synaptic loss in a reconstructed cortical-hippocampal network. a. Phase contrast picture of the microfluidic device comprising funnel-shaped micro-channels allowing unidirectional axonal growth. Cortical neurons (CX) were seeded in the left chamber, hippocampal neurons $(\mathrm{Hi})$ in the right chamber, and were cultured for 14 days to reconstruct a cortical-hippocampal network. A cartoon representing one cortical neuron connected to one hippocampal neuron is inserted for clarity. $\mathbf{b}$-e. Effect of A 42 oligomers and A $25-35$ peptides on synaptic connections. Cortical and hippocampal neurons were cultured in $\mu F D$ chambers as shown in a. b,c)

Representative fluorescence micrographs from somato-dendritic compartment of Cx neurons (left panels) and from Hi neurons in the distal chamber receiving cortical fibers (right panels). Cx chambers were treated with sham (b, $\varnothing / \varnothing)$ or A $325-35$ (c, A $\beta / \varnothing)$. Neurons, axons and synapses were immunodetected using anti-MAP2 (blue), anti-a-tubulin (green), and anti-a-synuclein (red) respectively. Scale bar: 20 um. Similar presynaptic patterns were observed with anti-synaptophysin labeling (not shown). d) Representative higher magnification showing presynaptic clusters (anti-a-synuclein, red) affixed to hippocampal dendrites (anti-MAP2, blue) in hippocampal neurons cultured alone (Hi) connected as in b $(H i-C X)$ and connected plus treated with $A \beta$ as in $\mathbf{c}(H i-C x+A \beta)$. e) Quantification of presynaptic structures affixed to hippocampal dendrites

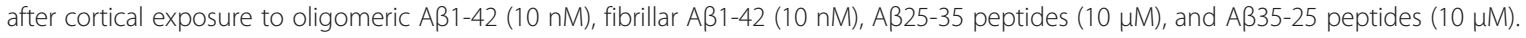
$\left(n=3 ;{ }^{* *} \mathrm{P}<0.01 ;{ }^{* * *} \mathrm{P}<0.001\right.$, ANOVA $)$

on direct contact but also on the location of its subcellular deposition. Axons are relatively resistant to direct $A \beta$ exposure, which is in line with our recent observation showing that axonal endings are resistant to direct proapoptotic insults [6]. Our observation with JNK and caspase pharmacological inhibitors suggest that both enzyme families are implicated locally (within axon) in the process of axonal degeneration, as already observed with other neuronal death inducers [6]. We also shows, for the first time, that axonal addition of $\mathrm{NAD}^{+}$is protective against $A \beta$-peptide induced axonal degeneration (Figure 2). Since $\mathrm{NAD}^{+}$is a well-established axo-protectant in the context of Wallerian degeneration, this raises the question whether there might be a $\mathrm{NAD}_{+}$controlled molecular pathway implicated in both $A \beta$-induced axon degeneration and Wallerian degeneration. Therefore $\mathrm{NAD}^{+}$related 

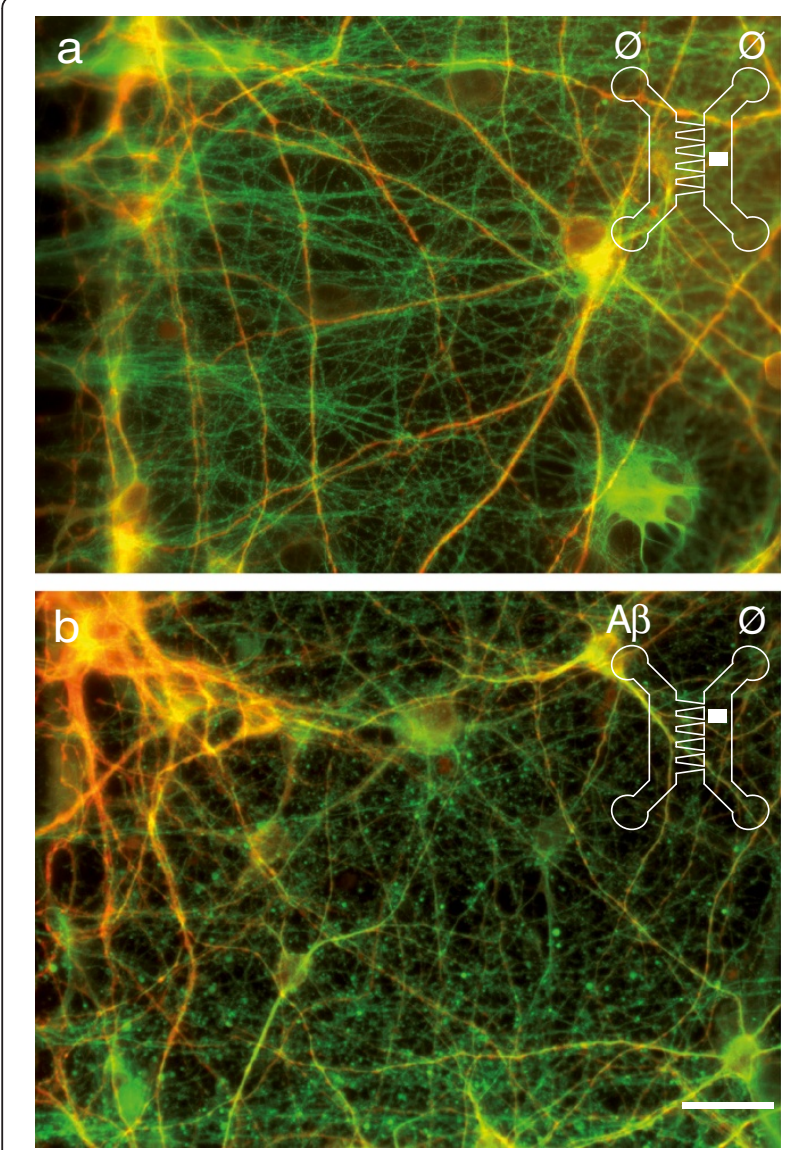

Figure 4 Exposure of cortical somata to $A \beta$-peptide induces an axonal loss in a reconstructed cortical-hippocampal network. Cortical (CX) and hippocampal ( $\mathrm{Hi})$ neurons were cultured for 14 days in $\mu F D$ chambers as in Figure 2. Hippocampal neurons and projecting cortical axons were immunodetected using anti-MAP2 (red), anti-a-tubulin (green). Cx chambers were treated with sham $(\mathbf{a}, \varnothing / \varnothing)$ or $10 \mu \mathrm{M} A \beta 42$ oligomers (b, A $/ \varnothing)$ for 48 hours.

Representative fluorescence micrographs from the distal hippocampal chamber receiving cortical fibers. Scale bar: $20 \mu \mathrm{m}$.

pathways might offer interesting targets to slow down dying back induced processes.

In some $A D$ patients, increased $A \beta$ is associated with complex disturbances of neuronal activity (e.g. epileptic activity) [15] and neurotransmission dysfunctions have been described in early phases of AD models $[16,17]$. Such local circuit disturbance might potentially lead to a broader network disruption in remote areas through neuronal projections. Interestingly, we observed that a mild somatic glutamatergic stress exacerbates distant axonal toxicity of $A \beta$. This suggests that cumulative and multi-focal stresses might play an important role in disease progression, by switching from local (and probably recoverable) minor dysfunctions to extended neuronal alterations like permanent synaptic, axonal or even cell bodies loss. Several non-exclusive mechanisms may account for AD-related spatiotemporal progression in the brain. This may involve neurotrophic factors withdrawal, aberrant neurotransmission, synaptic loss, excitotoxicity and prion-like spread of pathogenic misfolded proteins like trans-synaptic spread of $A \beta$ [3]. Interestingly, in our paradigm, blocking NMDA receptors on hippocampal neurons during cortical somato-dendritic $A \beta$ treatment prevented Tau Thr231 phosphorylation. These results are consistent with studies reporting that $A \beta$ could potentiate potassium-evoked glutamate release from neurons $[18,19]$.

\section{Conclusion}

While brain complexity, with its interconnected neuronal loops, complicates the in vivo analysis of pathophysiological initiation and spreading mechanisms, we were able for the first time to evaluate the distant effects of local cortical $\beta$-amyloid deposits on neuronal subcompartments and networks in $\mu \mathrm{FD}$-based reconstructed cortico-hippocampal networks. We show that a strictly local somato-dendritic amyloid trigger is sufficient to recapitulate a dying-back process, and to initiate an oriented neuron-to-neuron progression of pathological events. A $\beta$ peptide accumulation in the somato-dendritic compartment of cortical neurons leads to a fast anterograde propagation of degenerative signals toward endings, resulting in presynaptic collapse. This fast loss of cortical presynapses is associated with early trans-synaptic dysfunction such as NMDAR-dependent tau phosphorylation in postsynaptic hippocampal neurons. Hence, reconstructed cortico-hippocampal $\mu$ FD networks offer a new tool to decipher mechanisms that could underlie dying back and Braak's staging.

\section{Methods}

\section{Primary culture in microfluidic chips}

Microfluidic chips were realized as described in [8]. The design used for network reconstruction comprises two culture chambers each connected to two reservoirs and separated by a series of $500 \mu \mathrm{m}$-long asymmetrical micro-channels ( $3 \mu \mathrm{m}$ high, tapering from $15 \mu \mathrm{m}$ to $3 \mu \mathrm{m}$ ) [8]. E16 embryos (Swiss mice, Janvier, France) were micro-dissected in GBSS (without $\mathrm{CaCl}_{2}$ and $\mathrm{MgCl}_{2}$ ) $+0.1 \%$ glucose (Merck), digested with papain $(20 \mathrm{U} / \mathrm{mL})$ and mechanically dissociated in DMEM containing DNAse. $120.10^{3}$ cortical cells and $45.10^{3}$ hippocampal cells were seeded in the chamber on contact with the wide and narrow sides of micro-channels, respectively. Cells were cultured in DMEM glutamax (Invitrogen) supplemented with 10\% FBS (PAA), N2, streptomycin/penicillin and B27 (Invitrogen). The culture medium was renewed every 3-5 days. Cortical axon entered the micro-channels and reached the hippocampi-containing chamber in 4-5 days. The cortico-hippocampal oriented network was maintained routinely up to $2-3$ weeks in vitro. 

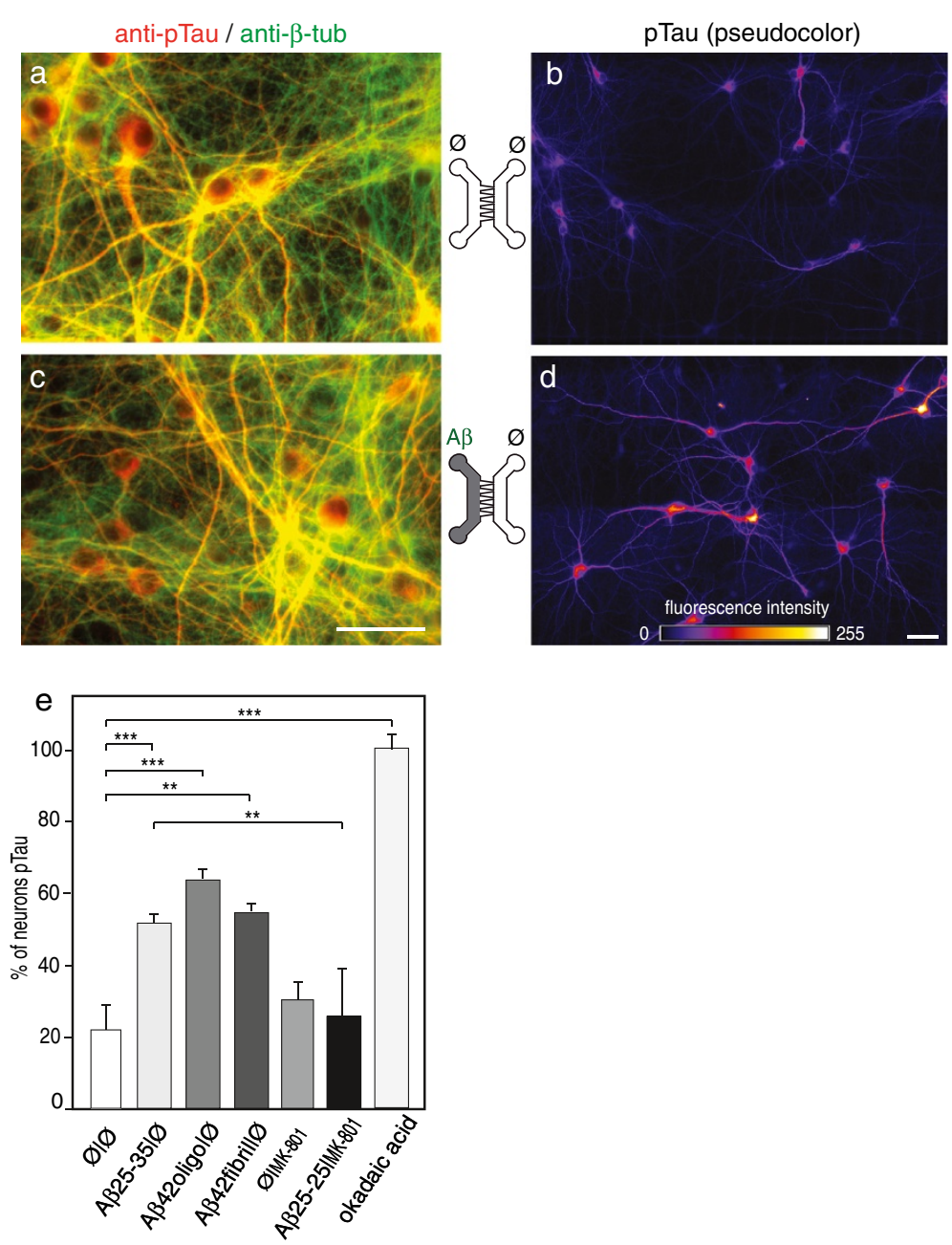

Figure 5 Cortical $A \beta$ peptide deposition induces glutamate-dependent hippocampal Tau phosphorylation. Cortical $(C x)$ and hippocampal (Hi) neurons were cultured in $\mu F D$ chambers as in Figure 2a. a,c. Representative fluorescence micrograph ofcortical neurons (left $\mu F D$ chamber) after immunostaining of phosphorylated Tau (pTau Thr231, red; $\beta$-tubulin, green). Effect of A $\beta 42$ oligomers and A $325-35$ peptides on synaptic connections. Cortical and hippocampal neurons were cultured in $\mu \mathrm{FD}$ chambers as shown in $\mathrm{a}$. b,c) Representative fluorescence micrographs from somato-dendritic compartment of Cx neurons (left panels) and from Hi neurons in the distal chamber receiving cortical fibers (right panels). $\mathbf{b}, \mathbf{d}$. Representative fluorescence micrograph of hippocampal neurons receiving cortical axons after immunostaining of pTau (Thr231; pseudo-colors). b) Control conditions $(\varnothing / \varnothing)$, d) cortical treatment with $A \beta 25-35(10 \mathrm{nM})$ for $24 \mathrm{~h}$. Scale bar: $20 \mu \mathrm{m}$. e. Quantification of pTau-positive hippocampal neurons after $24 \mathrm{~h}$ cortical exposure to fibrillar A $325-35$, oligomeric A 42 or fibrillar A 42 (10 nM, each) in the presence or absence of the NMDAR antagonist MK801 (10 $\mu \mathrm{M})$. The results were compared to pTau-positive neurons induced by exposure of Hi neurons to okadaic acid (1 nM, $24 \mathrm{~h}$ ) $\left(n=3{ }^{* * P}<0.01 ; * * P<0.001\right.$, ANOVA).

\section{Oligomeric and fibrillar $A \beta$ peptide preparations}

Oligomeric and fibrillar forms of A $\beta 1-42$ (Tocris Bioscience, MN, USA), were produced according to [20] and controlled by electron microscopy (Additional file 2: Figure S2). Briefly, lyophilized peptides were solubilized at $1 \mathrm{mM}$ in $1,1,1,3$, 3, 3,-hexafluoro-2-propanol (HFIP, Sigma Aldrich). After 30 min of incubation at RT, HFIP was evaporated overnight and peptides were dried (Speed Vac, $1 \mathrm{~h} 4^{\circ} \mathrm{C}$ ). Then, $\mathrm{A} \beta$ peptide stock solution were obtained byresolubilizationet $5 \mathrm{mM}$ in dimethylsulfoxide (DMSO, Sigma Aldrich). To obtain oligomers, A $\beta$ stock solution was diluted at $100 \mu \mathrm{M}$ in phenol-free DMEM-F12 medium (Life technologies), incubated $24 \mathrm{~h}$ at $4^{\circ} \mathrm{C}$ and centrifuged at $20000 \mathrm{~g}(10 \mathrm{~min}$; $4^{\circ} \mathrm{C}$ ) before supernatant (soluble $\mathrm{A} \beta$ fraction) collection. To obtain fibrils, $\mathrm{A} \beta$ stock solution was diluted at $100 \mu \mathrm{M}$ in $\mathrm{HCl} 10 \mathrm{mM}$ and then incubated at $4^{\circ} \mathrm{C}$ for $24 \mathrm{~h}$.

\section{Electron microscopy}

A $\beta$ sample aliquots $(10 \mu \mathrm{M})$ were allowed to adsorb onto carbon-coated 200-mesh copper grids (EMS, PA, USA) for 2 minutes before blotting off. Then, grids were incubated for 45 seconds in uranyl acetate $2.5 \%(\mathrm{w} / \mathrm{v})$ to 


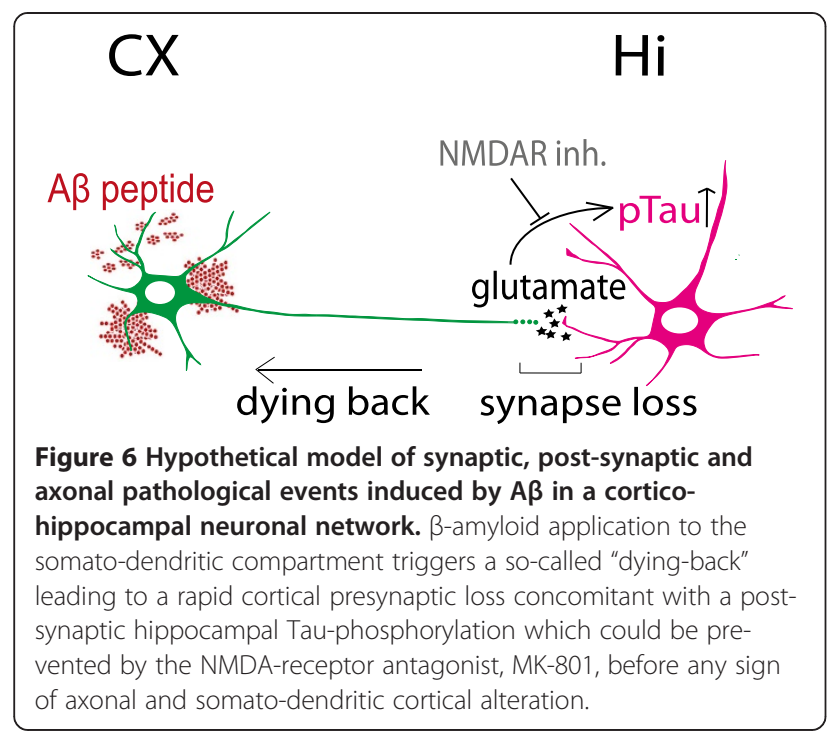

produce a negatively stained protein loaded grid. Images were recorded on a Zeiss 912 omega electron microscope (Carl Zeiss Group, Oberkochen, Germany).

\section{Treatments}

The following compounds were used: $A \beta 1-42$ peptide (1428, Tocris), A $325-35$ peptide (H-1192, Bachem), control A $335-25$ peptide (H2964, Bachem), glutamate (G8415, Sigma), MK-801 (0924, Tocris), $5 \mathrm{mM} \mathrm{NAD}^{+}$ (Sigma), $50 \mu \mathrm{M}$ z-VAD-fmk (R\&D Systems, Minneapolis, MN, USA) and $50 \mu$ M SP 600125 (Sigma). Oligomeric and fibrillar forms of A $\beta 1-42$ were produced according to [20] and controlled by electron microscopy. To ensure fluidic isolation between compartments, a hydrostatic pressure difference was generated by over-pressurizing the non-treated chamber as described in [21].

\section{Immunofluorescence detection}

Cultures were fixed (4\% PFA, 20 min RT) and immunostained as described in [13]. Primary antibodies included anti- $\alpha$-tubulin-FITC (F2168, sigma), anti-MAP2 (M4403, Sigma), anti-Synaptophysin (S5768, Sigma), anti-Vglut1, anti- $\alpha$-synuclein (4179, cell signaling), anti-pTau Thr231 (44746G, Invitrogen). Species-specific secondary antibodies (coupled to Alexa 350, 488, 555) were used (Invitrogen). Images were acquired with an Axio-observer Z1 (Zeiss) fitted with a cooled CCD camera (CoolsnapHQ2, Ropert Scientific) and images were analyzed using ImageJ.

\section{Quantification of axonal fragmentation}

For axonal fragmentation analysis and quantification we used fluorescence microscopy (immuno-detection of tubulin), phase contrast, and picture analysis according to a previously described protocol [22]. Briefly, we used a macro developed in NIH ImageJ software that utilizes the Otsu thresholding algorithm, and a particle analyzer algorithm of ImageJ. The total area of axonal regions with circularity greater than 0.9 was determined and normalized by the total axonal area, which was measured from the thresholded image. This ratio, termed fragmentation index, is an indicator of the average axonal fragmentation level and is used in statistical comparisons. Indices of $0.005,0.083$, and 0.157 correspond to $<5 \%, 50 \%$, and $>95 \%$ fragmentation, respectively.

\section{Quantification of synapse loss}

Synaptic disconnection was assessed through fluorescence microscopy by counting $\alpha$-synuclein clusters affixed to MAP2 positive hippocampal dendrites. Images were all obtained with the same acquisition parameters. The images were similarly processed with ImageJ software before being used for quantification: the brightness/contrast of all control images was optimized manually to eliminate the background and to maximize the signal. The means of the minimum and maximum intensities were then calculated in the control condition, after which these settings were applied to all images. The images of the three $\alpha$-synuclein/ $\alpha$-Tubulin/ MAP2 stainings were merged and the resulting image was used to define the zone where hippocampal dendrites were sufficiently innervated by cortical fibres. $\alpha$-synuclein/MAP2 merges were then used for quantification.

\section{Quantification of Tau phosphorylation}

Tau phosphorylation was assessed by counting the number of neurons presenting pTau levels above a fixed threshold. Images were all obtained using the same acquisition parameters. The images were similarly processed with ImageJ software before being used for quantification: the brightness/ contrast of all control images was optimized manually to eliminate the background and to maximize the signal. The means of the minimum and maximum intensities were then calculated in the control condition, after which these settings were applied to all images. All pTau images were then processed with the "Lookup Tables, fire" plugin to visualize the intensity of pTau staining with pseudo-colours. The number of neurons above a fixed colour threshold was then counted and normalized by the total number of neurons to have the percentage of hyperphosphorylated tau neurons.

\section{Statistical analysis}

Differences were assessed by ANOVA, followed, when appropriate, by a post-hoc Bonferoni test. For all analysis * -value $<0.05$; * p-value $<0.01$; *** p-value $<0.001$.

\section{Additional files}

Additional file 1: Figure S1. Dendritic morphology of cortical neurons before and after $A \beta$ peptide treatment. Cortical $(C X)$ neurons were cultured for 14 days in $\mu F D$ chambers as in Figure 1. Dendrites and 
pre-synaptic clusters of cortical neurons were immuno-detected using anti-MAP2 (blue), anti-VGLUT1 (red). Cx chambers were treated with sham $(\mathbf{a}, \varnothing / \varnothing)$ or $10 \mu \mathrm{M} A \beta 42$ oligomers $(\mathbf{b}, A \beta / \varnothing)$ for 48 hours. Representative fluorescence micrographs of the cortical chamber are shown. Scale bar: $20 \mu \mathrm{m}$

Additional file 2: Figure S2. $A \beta$ morphology analysed by electron microscopy. Negative stain of TEM images of either A $\beta 1-42$ oligomers (a) or fibrils (b) and A $325-35$ aggregates (c)

\section{Competing interests}

The authors declare that they have no competing interests.

\section{Acknowledgments}

This work was supported by "Foundation plan Alzheimer 2010-13", ANR

"PrionSensiTNF: Blan-1312-02" 2011-14 and ANR "Neuroscreen:

2011-RPIB-008-01" 2012-16. B.D. was funded by an MNRT fellowship.

\section{Author details}

Institut de Biologie Paris Seine (IBPS), 75005 Paris, France. ${ }^{2}$ CNRS UMR 8256 Biological Adaptation and Ageing, Team 5: Degenerative Processes in Neurons and Networks, F-75005 Paris, France. ${ }^{3}$ CNRS UMR 7102 Neurobiologie des Processus Adaptatifs, F-75005 Paris, France. ${ }^{4}$ Sorbonne Universités, UPMC Université Paris 6, F-75005 Paris, France. ${ }^{5}$ Institut Curie, Laboratory of Macromolecules and Microsystems in Biology and Medicine, F-75005 Paris, France. ${ }^{6}$ CNRS UMR 168, F-75005 Paris, France. Institute of Reproductive and Developmental Biology, Imperial College London, Hammersmith Hospital, London W12 ONN, UK.

Received: 16 August 2014 Accepted: 14 September 2014

Published online: 25 September 2014

\section{References}

1. Braak H, Alafuzoff I, Arzberger T, Kretzschmar H, Del Tredici K (2006) Staging of Alzheimer disease-associated neurofibrillary pathology using paraffin sections and immunocytochemistry. Acta Neuropathol 112(4):389-404

2. Thal DR, Rüb U, Orantes M, Braak H (2002) Phases of A beta-deposition in the human brain and its relevance for the development of AD. Neurology 58(12):1791-1800

3. Warren JD, Rohrer JD, Schott JM, Fox NC, Hardy J, Rossor MN (2013) Molecular nexopathies: a new paradigm of neurodegenerative disease Trends Neurosci 36(10):561-569

4. Terry RD (2000) Cell death or synaptic loss in Alzheimer disease. J Neuropathol Exp Neurol 59(12):1118-1119

5. Scheff SW, Price DA, Schmitt FA, DeKosky ST, Mufson EJ (2007) Synaptic alterations in CA1 in mild Alzheimer disease and mild cognitive impairment. Neurology 68(18):1501-1508

6. Magnifico S, Saias L, Deleglise B, Duplus E, Kilinc D, Miquel MC, Viovy UL, Brugg B, Peyrin JM (2013) NAD + acts on mitochondrial SirT3 to prevent axonal caspase activation and axonal degeneration. FASEB J 27(12):4712-4722

7. Millet LJ, Gillette MU (2013) New perspectives on neuronal development via microfluidic environments. Trends Neurosci 35(12):752-761

8. Peyrin JM, Deleglise B, Saias L, Vignes $M$, Gougis P, Magnifico S, Betuing S, Pietri M, Caboche J, Vanhoutte P, Viovy JL, Brugg B (2011) Axon diodes for the reconstruction of oriented neuronal networks in microfluidic chambers, Lab Chi 11(21):3663-3673

9. Mairet-Coello G, Courchet J, Pieraut S, Courchet V, Maximov A, Polleux F (2013) The CAMKK2-AMPK kinase pathway mediates the synaptotoxic effects of Abeta oligomers through Tau phosphorylation. Neuron 78(1):94-108

10. Luna-Munoz J, Chávez-Macías L, García-Sierra F, Mena R (2007) Earliest stages of tau conformational changes are related to the appearance of a sequence of specific phospho-dependent tau epitopes in Alzheimer's disease. J Alzheimers Dis 12(4):365-375

11. Jucker M, Walker LC (2013) Self-propagation of pathogenic protein aggregates in neurodegenerative diseases. Nature 501(7465):45-51

12. Song HL, Shim S, Kim DH, Won SH, Joo S, Kim S, Jeon NL, Yoon SY (2014) beta-amyloid is transmitted via neuronal connections along axonal membranes. Ann Neurol 75(1):88-97

13. Deleglise B, Lassus B, Soubeyre V, Alleaume-Butaux A, Hjorth JJ, Vignes M, Schneider B, Brugg B, Viovy JL, Peyrin JM (2013) Synapto-protective drugs evaluation in reconstructed neuronal network. PLoS One 8(8):e71103
14. Adalbert R, Coleman MP (2013) Axon pathology in age-related neurodegenerative disorders. Neuropathol Appl Neurobiol 39(2):90-108

15. Palop JJ, Mucke L (2009) Epilepsy and cognitive impairments in Alzheimer disease. Arch Neurol 66(4):435-440

16. Palop JJ, Chin J, Roberson ED, Wang J, Thwin MT, Bien-Ly N, Yoo J, Ho KO, Yu GQ, Kreitzer A, Finkbeiner S, Noebels JL, Mucke L (2007) Aberrant excitatory neuronal activity and compensatory remodeling of inhibitory hippocampal circuits in mouse models of Alzheimer's disease. Neuron 55(5):697-711

17. Palop JJ, Mucke L (2010) Amyloid-beta-induced neuronal dysfunction in Alzheimer's disease: from synapses toward neural networks. Nat Neurosc 13(7):812-818

18. Kabogo D, Rauw G, Amritraj A, Baker G, Kar S (2008) ss-amyloid-related peptides potentiate $\mathrm{K}+-$ evoked glutamate release from adult rat hippocampal slices. Neurobiol Aging 31(7):1164-1172

19. Liang Z, Liu F, labal K, Grundke-lqbal I, Gong CX (2009) Dysregulation of tau phosphorylation in mouse brain during excitotoxic damage. J Alzheimers Dis 17(3):531-539

20. Stine WB, Dahlgren KN, Krafft GA, LaDu MJ (2003) In vitro characterization of conditions for amyloid-beta peptide oligomerization and fibrillogenesis. J Biol Chem 278(13):11612-11622

21. Taylor AM, Blurton-Jones M, Rhee SW, Cribbs DH, Cotman CW, Jeon NL (2005) A microfluidic culture platform for CNS axonal injury, regeneration and transport. Nat Methods 2(8):599-605

22. Kilinc D, Peyrin JM, Soubeyre V, Magnifico S, Saias L, Viovy JL, Brugg B (2011) Wallerian-like degeneration of central neurons after synchronized and geometrically registered mass axotomy in a three-compartmental microfluidic chi. Neurotox Res 19(1):149-161

doi:10.1186/s40478-014-0145-3

Cite this article as: Deleglise et al:: $\beta$-amyloid induces a dying-back process and remote trans-synaptic alterations in a microfluidic-based reconstructed neuronal network. Acta Neuropathologica Communications 2014 2:145.

\section{Submit your next manuscript to BioMed Central and take full advantage of:}

- Convenient online submission

- Thorough peer review

- No space constraints or color figure charges

- Immediate publication on acceptance

- Inclusion in PubMed, CAS, Scopus and Google Scholar

- Research which is freely available for redistribution

Submit your manuscript at www.biomedcentral.com/submit
C Biomed Central 